
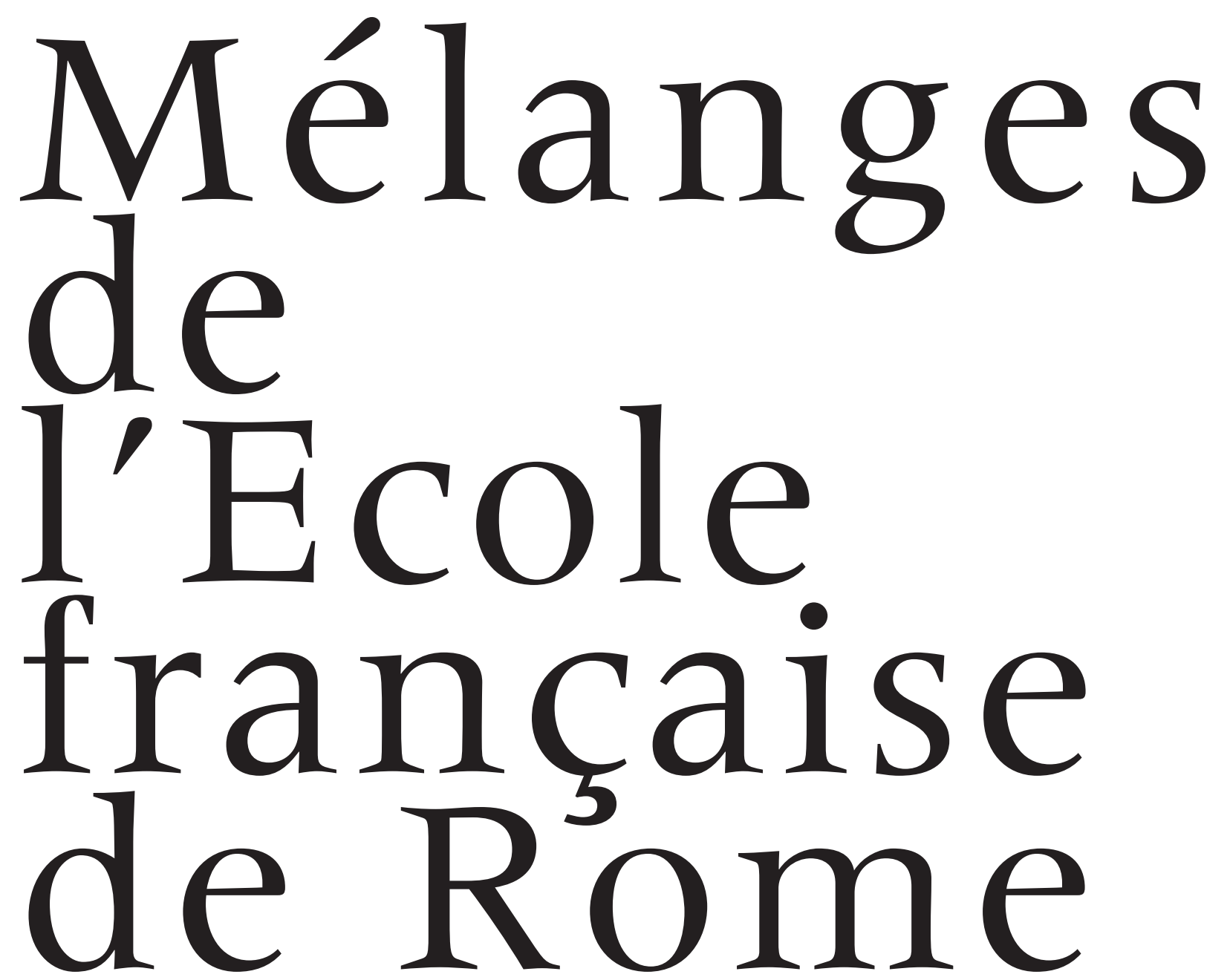

Moyen Âge

MEFRM

132-2 2020 


\title{
Nichil solvit
}

\section{Norma e pratica nella contribuzione ecclesiastica per la monarchia nella Corona d'Aragona durante il papato di Avignone e lo Scisma (1309-1418)}

\author{
Esther Tello HernándeZ
}

Escuela Española de Historia y Arqueología de Roma. EEHAR-CSIC. Roma - esther.tello@eehar.csic.es

\begin{abstract}
Il presente studio ha lo scopo di sistematizzare le varie forme di contribuzione ecclesiastica a favore dei sovrani della Corona d'Aragona durante il basso Medioevo. Non c'è dubbio che gli ecclesiastici, così come ogni altro stamento o gruppo sociale, subissero le pressanti richieste della monarchia quando si trattava di partecipare alle contribuzioni fiscali. Molte di esse erano contemplate nella legislazione canonica e civile; altre, invece, si situavano al limite delle stesse e, in alcuni casi, addirittura travalicavano la normativa. Questa realtà crescente si osserva perfettamente nella Corona d'Aragona durante il XIV secolo e agli inizi del XV, un momento in cui la contribuzione della Chiesa raggiunse il suo culmine rispetto ai periodi anteriori.
\end{abstract}

Parole chiave: Corona d'Aragona, papato di Avignone, fiscalità ecclesiastica, trattativa.

\begin{abstract}
The present study aims to analyze the different ways of ecclesiastical contribution to the king in the Crown of Aragon during the late Middle Ages. Church, like every other social group or state, was pressed by the monarchy in order to participate in the tax demands. Many of those contributions were included in regulations established by the papacy or by the civil legislation; others, however, were at the limit of these regulations or, in some cases, exceeded them. This increasing reality is perfectly observed in the Crown of Aragon during the $14^{\text {th }}$ century and at the beginning of the $15^{\text {th }}$ century, a time when the contribution of the Church overreached the limits set by previous periods.
\end{abstract}

Keyword: Crown of Aragon, papacy of Avignon, ecclesiastical taxation, negotiation.

\section{INTRODUZIONE}

${ }^{1}$ Il presente articolo analizza la norma e la pratica della contribuzione ecclesiastica rispetto

1. Questo lavoro è stato possibile grazie a un contratto di postdottorato presso la Scuola Spagnola di Storia e Archeologia di Roma (EEHAR-CSIC). Inoltre è stato sviluppato nel quadro del progetto del Ministero spagnolo "La desigualdad económica en las ciudades catalanas y mallorquinas durante la baja Edad Media» e del gruppo di ricerca della Generalitat di Catalogna «Renda feudal i fiscalitat a la Catalunya baixmedieval» (2017 SGR 1068). Abbreviazioni utilizzate: $\mathrm{ACA}=$ Archivio della Corona d'Aragona; $\mathrm{RP}=$ Reale Patrimonio; $\mathrm{MR}=$ Maestro Razionale; $\mathrm{TR}=$ Tesoro Regio; Can = Reale Cancelleria; $\mathrm{ASV}=$ Archivio Secreto Vaticano; Reg. Vat = Registro Vaticano; Reg. Aven = Registro Avignonese. Gli importi monetari sono espressi in «libras», "sueldos» e «dineros» barcellonesi (l.b., s.b., d.b.), aragonesi (l.j., s.j., d.j.) e fiorini d'Aragona (fl.). alle richieste della monarchia aragonese durante il XIV e il XV secolo, utilizzando documenti normativi e di archivio conservati presso l'Archivio della Corona d'Aragona e l'Archivio Segreto Vaticano. Negli ultimi tempi, tali questioni hanno suscitato l'interesse di numerosi studiosi, i quali si sono dedicati all'analisi del discorso e della prassi utilizzati per legittimare e giustificare il tributo di fronte alla Chiesa. In particolare, dobbiamo tenere presente come, dalla metà del XIII secolo, e per tutto il XIV, si verificò un profondo cambiamento nelle strutture fiscali e finanziarie dell'Occidente medievale. La monarchia cercò nuove risorse affinché le casse reali diventassero più ricche e uno dei modi per ottenerle fu il ricorso all'aiuto del clero. A tal fine, nella cerchia intellettuale dei monarchi furono elaborate diverse argomentazioni tese a giustifi- 
care i contributi richiesti agli ecclesiastici. ${ }^{2}$ Anche nella Penisola iberica il tema è stato affrontato sotto diversi punti di vista, benché vada sottolineato come per la Corona d'Aragona manchi ancora un solido bagaglio di studi sulla materia. ${ }^{3} \mathrm{Di}$ conseguenza, riteniamo sia necessario continuare ad analizzare la diatriba sorta tra la monarchia e la Chiesa intorno al tributo fiscale nella Corona durante il basso Medioevo, dall'inizio del papato di Avignone fino all'inizio del cosiddetto Grande Scisma d'Occidente. ${ }^{4}$

In genere, durante il XIV secolo, osserviamo come le risorse materiali a cui poteva ricorrere la monarchia aragonese erano insufficienti, soprattutto quando doveva far fronte a grandi imprese militari. Ciò spiega la continua ricerca di finanziamento da parte dei regnanti e la conseguente imposizione di numerosi gravami ${ }^{5}$. Tale dinamica provocò una pressione fiscale ininterrotta che colpì prima le grandi e piccole città soggette ai tributi e ai diritti regi, per estendersi poi agli ambiti signorili ed ecclesiastici. Detto in altro modo, similmente a quanto accadde in altre monarchie occidentali, anche nella Corona d'Aragona assistiamo all'ampliamento degli ambienti soggetti alla pressione fiscale oltre gli stretti confini del patrimonio reale, fino ad arrivare a tutti i sudditi. ${ }^{6}$

D'altra parte, fin dal XII-XIII secolo, nella curia pontificia si avviarono riforme amministrative che posero le basi di quanto accadde nei secoli successivi. La Chiesa, come organismo istituzionale, stava consolidando il suo potere politico ed economico. In generale, il suo valore si basava su tre principi

2. Genet - Le Page - Mattéoni 2017, e in particolare Hébert 2017, p. 11-40; Barralis - Genet 2014, e in particolare, Davis 2014, p. 25-33; Ormrod - Killick - Bardford 2017; Giannini 2014; Isenmann 2010; Lecuppre 2010; Scordia 2005, p. 107-148; Kempshall 1999.

3. Per un'introduzione a questo argomento nella Corona d'Aragona citiamo, tra gli altri, Morelló 2018, p. 177-210; 2013a; 2013b, p. 169-279; Verdés 2013, p. 131-168; Menjot - Sánchez 2011, Sabaté 1998, p. 757-788. Per quanto riguarda Castiglia vedi Ortego 2012, p. 145-176; 2009, p. 113-138; Carretero 2013, p. 79-104; Fortea 2015, p. $473-508$.

4. Il nostro scopo non è quello di fare riferimento alle somme finali raccolte, né ai calcoli percentuali totali. Per realizzare uno studio in tal senso, infatti, dovremmo sviluppare un lavoro molto più ampio, tenendo conto di molte variabili: Morelló 2017; Tello 2017.

5. Bonney 1994, 1999.

6. Come opere di sintesi citiamo fra tutte Ladero Quesada et al. 2015; Sánchez - Furio - Sesma 2008, p. 99-130. fondamentali: l'esistenza di una particolare giurisdizione ecclesiastica, il riconoscimento dell'esenzione fiscale e il diritto a ricevere redditi di varia natura da religiosi e fedeli provenienti da diversi territori europei. In particolare, risalta il consolidamento di diverse circoscrizioni territoriali in tutta l'Europa cristiana, circostanza che permise alla Santa Sede, attraverso i propri rappresentanti, di esercitare un maggior controllo su tali aree. ${ }^{7}$ Pertanto, così come le monarchie costruirono i propri stati feudali, anche la Chiesa consolidò il suo potere politico ed economico. Durante tutto il XIV secolo si documenta uno sviluppo della fiscalità pontificia come conseguenza delle sempre maggiori necessità della Camera Apostolica, principale organo finanziario dell'erario papale. Com'è noto, tale circostanza incise sulle relazioni tra il papato e i principi cristiani in quanto, con sempre maggiore frequenza, sostanziose risorse fiscali vennero trasferite dai vari territori della Cristianità alle casse pontificie ma, nel contempo, parte di tali esazioni destinate alla Sede Apostolica furono captate dai sovrani. ${ }^{8}$

In tale contesto, con il proposito di ricostruire la strategia fiscale della Corona aragonese nei confronti degli ecclesiastici durante questo periodo, vogliamo insistere su due questioni fondamentali. Da una parte, ci interessa sapere in che modo veniva giustificata la richiesta di tassazione agli ecclesiastici da parte della monarchia e dei poteri laici. Dall'altra, risulta altrettanto interessante indagare la posizione adottata dalla Chiesa di fronte alle richieste dei sovrani. Le istituzioni ecclesiastiche, infatti, tentarono di conciliare la necessaria e ineludibile contribuzione ad alcune richieste fiscali con le irrinunciabili libertà proprie del gruppo ecclesiastico.

In considerazione di ciò, articoliamo il presente studio in tre parti. In primo luogo, definiremo

7. Tra i molti studi che affrontano la configurazione territoriale e fiscale del papato, ci riferiamo a Favier 1966; Lunt 1943 (1965), p. 3-56; Cevins - Matz 2010, in particolare, è suggestiva la sezione che si riferisce agli aspetti sociologici del clero e dei laici. Per la Corona d'Aragona: Morelló 2009, p. 67-125.

8. In generale, questa evoluzione dell'amministrazione papale è da intendersi nel contesto di un'intensa attività politica e militare: guerre in Italia, la guerra dei Cent'anni, il mantenimento stesso della corte papale, lo Scisma. Su tali questioni: Payan 2010; Millet 2010, 2013; Souza Bayona 2013; Jamme 2005. 
brevemente l'ordinamento legale che era alla base della teorica immunità ecclesiastica, in generale, e il suo riflesso nella Corona d'Aragona, in particolare. Quindi, analizzeremo i limiti dell'esenzione ecclesiastica, ossia le diverse forme di pressione volte a ottenere la partecipazione della Chiesa ad alcune delle principali imposizioni fiscali stabilite dai sovrani aragonesi. In secondo luogo, ci concentreremo sui diversi modi di contribuzione effettiva della Chiesa aragonese realizzata secondo l'ordinaria prassi fiscale. Infine, illustreremo alcuni casi di partecipazione coatta alle contribuzioni, quando, cioè, queste venivano prelevate senza il consenso degli ecclesiastici (o dei loro superiori), superando la cosiddetta forma di contribuzione ortodossa/ abituale, ovvero quella che, grossomodo, rispettava i parametri stabiliti dalla norma.

\section{L'ORDINAMENTO LEGALE \\ DELLA CONTRIBUZIONE ECCLESIASTICA \\ NELLA CORONA D'ARAGONA}

Così come accadeva in altre latitudini, gli ecclesiastici costituivano un importante gruppo sociale nelle città e nelle cittadine della Corona d'Aragona durante il basso Medioevo. Secondo i dati disponibili, nella Barcellona del basso Medioevo, per esempio, gli ecclesiastici costituivano circa l' $8-10 \%$ del totale della popolazione, e anche in altri luoghi della Corona la percentuale si attestava tra il 5 e il $10 \%$ circa. ${ }^{9}$ Il clero si distingueva dalla popolazione laica e formava un gruppo particolare che godeva di uno statuto giuridico proprio e, per quanto qui $c^{\prime}$ interessa, di esenzioni fiscali. La risposta del clero alle richieste fiscali del potere laico permette di definire, in gran misura, l'identità dell'eterogeneo gruppo ecclesiastico, la cui esenzione effettiva, però, variò in base alle circostanze del momento e alla condizione religiosa dei chierici. ${ }^{10}$

A tale proposito ricordiamo che tradizionalmente il clero è stato considerato un soggetto fiscale che non doveva contribuire alle esazioni imposte dai laici. Ciononostante, a partire dal XIII

9. Baucells 2004-2007, vol. III, p. 2277-2292; Morelló 1999, p. 692.

10. Per una disamina sugli ecclesiastici secolari intesi come gruppo sociale nel contesto catalano: Sabaté 1998, p. 757-788; Morelló 1999, p. 689-729; 2005; Bertran 1990, p. 66-69. secolo si contemplò che, in alcune circostanze concrete, le autorità laiche potessero sollecitare la contribuzione degli ecclesiastici, così come era stabilito nella normativa canonica e nel diritto civile. ${ }^{11}$ Ad esempio, nel IV Concilio lateranense del 1215, mediante il canone Adversus de immunitate ecclesiastica, si decretò che qualsiasi richiesta fiscale straordinaria doveva essere approvata dal pontefice e sarebbe stata autorizzata solo in caso di urgente necessità e in favore dell'utilità pubblica. ${ }^{12}$ Alla fine del XIII secolo, durante il papato di Bonifacio VIII, e nell'ambito delle tensioni con il re di Francia, vennero promulgate diverse bolle con le quali si affrontò il tema della partecipazione del clero alle imposizioni fiscali regie, mettendo in rilievo, almeno in teoria, la superiorità della Chiesa rispetto alla monarchia, e la necessità che la contribuzione fosse sempre pienamente libera e concessa solo a fronte di legittime esigenze della monarchia. ${ }^{13}$

Anche per quanto riguarda la Corona d'Aragona troviamo una produzione legislativa particolare che si concentra sui rapporti tra i religiosi e la monarchia. Ad esempio, Giacomo II stabilì nel 1316 e nel 1327 che gli ecclesiastici non dovevano contribuire alle imposizioni indirette se non secondo quanto disposto nella normativa e comunque solo in caso di estrema necessità. ${ }^{14}$ Quasi cento anni più tardi, nel 1413 e nell'ambito delle Cortes di Barcellona, Fernando I rispondeva a dei greuges, ovvero dei reclami presentati dal braccio ecclesiastico allo scopo di frenare l'intromissione della fiscalità regia nell'ambito ecclesiastico. Il monarca stabilì che, sebbene gli uomini di Chiesa fossero liberi ed esenti da numerose tasse ordinarie e indirette, i sovrani avevano il diritto di

11. Montagut 2004, p. 49-68; Verdés 2013, p. 133-134, 155.

12. Housley 1986, p. 63 (cf. Corpus Iuris Canonici, l. III, c. 49).

13. Questo è l'argomento principale del Clericis Laicos promulgato nel 1296 (cf. Corpus Iuris Canonici, l. III, c. 33, cap. 3). Tale disposizione prevedeva delle pene nel caso in cui un principe secolare avesse avanzato richieste contributive senza previa licenza papale. Così, fu stabilito che i sovrani e le autorità civili che avessero costretto i religiosi a pagare una tassa illecita sarebbero stati scomunicati. Lo stesso sarebbe accaduto agli ecclesiastici che avessero contribuito senza il permesso del Papa o a quelli implicati in altri casi di violazione delle libertà della Chiesa; Jamme 2015, p. 117-146.

14. Ad esempio, alla contribuzione erano tenuti gli ecclesiastici impegnati in attività economiche tipiche dei laici, quali l'artigianato, il commercio, ecc: Verdés 2013, p. 151. 
esigere certe contribuzioni, così come ormai accadeva da decenni. ${ }^{15}$

In definitiva, il clero era teoricamente esente e poteva contribuire solo in maniera volontaria e senza coercizione alcuna, così come era stabilito nella legislazione canonica generale e in quella civile della Corona d'Aragona. Orbene, la pressione fiscale e la creazione di una fiscalità generale nei diversi regni dell'Occidente europeo misero in discussione tale principio d'immunità teorica. Ciò testimonia come i limiti dell'esenzione ecclesiastica dipendevano, in realtà, da diversi fattori, quali le condizioni dell'erario regio, le relazioni tra i poteri civile e religioso, o le diverse categorie dei chierici sottoposti a contribuzione. Attraverso lo studio della pressione fiscale esercitata sul clero durante il basso Medioevo, possiamo osservare chiaramente il processo di progressiva erosione della supposta immunità degli ecclesiastici. Nel momento in cui ci approcciamo allo studio di queste diverse forme di contribuzione della Chiesa alle richieste del re, dobbiamo considerare anche, se non soprattutto, i numerosi conflitti documentati nel XIV secolo e nei primi decenni del $\mathrm{XV}$, vale a dire la conquista della Sardegna (1321-1324), la guerra di Granada (1329-1335), la guerra dello stretto di Gibilterra (1340) o la conquista di Maiorca (1343-1344). A questi, si aggiunsero, a partire dalla metà del secolo, altri due grandi scontri, fondamentali per capire tutto questo processo: le continue lotte per il dominio dell'isola di Sardegna, che durarono dal 1347 all'inizio del XV secolo, e la guerra contro il regno di Castiglia, che ebbe luogo tra il 1356 e il 1366, anche se le tensioni continuarono ancora per decenni e si tradussero in nuovi conflitti nel XV secolo, quando si sommarono di nuovo le campagne mediterranee e aumentarono le tensioni in tutta la Penisola iberica. ${ }^{16}$

15. Sánchez 2011, p. 133-167, in particolare p. 136-138 e p. 155-157. A seguito delle proteste degli ecclesiastici, furono redatti dei memoriali dove vennero raccolte le notizie di vari sussidi pagati dal clero e dai loro vassalli per quasi duecento anni. Lo scopo di questi memoriali era di chiarire come era andato mutando il contributo ecclesiastico e come si giustificavano le richieste non abituali.

16. Per un'introduzione generale sul contesto bellico in relazione alla pressione fiscale esercitata sul clero nella Corona d'Aragona: Morelló 2017, 2013; Menjot - Sánchez 2011.

\section{LA CONTRIBUZIONE ABITUALE}

In tale contesto, non risulta strano che sia proprio nel corso del XIV secolo che la contribuzione ecclesiastica verso i poteri laici è chiaramente attestata nei suoi diversi aspetti. In tutti questi casi, la natura del tributo sollecitato e la sua destinazione condizionavano la misura e le caratteristiche della riscossione. Inoltre, nonostante sia possibile parlare di una contribuzione abituale od ortodossa, e quindi legittima a norma di legge, si cercava di evitare la doppia contribuzione della Chiesa per un'identica tipologia d'imposta o per un medesimo scopo.

\section{La decima pontificia}

In primo luogo, osserviamo che un gran numero di religiosi (quelli che avevano ricevuto gli ordini e vivevano di rendite beneficiali) pagava numerose tasse pontificie che andavano direttamente nelle casse dei laici. La forma di contribuzione ecclesiastica più usuale fu la decima pontificia. Proclamata sempre dal papa e accettata dai chierici, la decima ebbe come finalità originaria quella di difendere la Terra Santa e, in generale, servire agli interessi della Santa Sede: pro oneribus romane ecclesiae. ${ }^{17}$ Tuttavia, a causa della situazione in cui si trovavano alcuni regni cristiani, come per esempio quelli della Penisola iberica, a partire dal XIII secolo la decima s'impiegò anche per far fronte alle guerre dell'Al-Andalus, di Granada e dell'Africa settentrionale. Nel nostro caso, durante tutto il XIV secolo, ma soprattutto nella seconda metà, la decima venne concessa anche ai sovrani aragonesi per far fronte alle spese necessarie per la conquista della Sardegna e alle rivolte dell'isola, che era feudo della Santa Sede e territorio vassallo del re d'Aragona. ${ }^{18}$

Questo tributo aveva, in principio, un carattere transitorio e straordinario, però man mano che ci addentriamo nel XIV secolo, il suo pagamento divenne un'entrata fiscale pressoché ordinaria della monarchia; infatti durante questo periodo, la Corona percepì i due terzi $\mathrm{o}$ addirittura l'intera

17. Favier 1966, p. 206-232; Lunt 1943 (1965), p. 71.

18. Maggiori informazioni a riguardo si possono reperire, oltre che nelle opere precedentemente citate, anche in Bertran 1997; Morelló 2011; Tello 2017, 2019. 
somma dell'imposta. Comunque sia, dal 1279 al 1456 furono vigenti almeno 21 decime pontificie in tutta la Corona d'Aragona; solo tra il 1349 e il 1410 ben 15 decime furono in vigore in tutta la Corona in maniera continua (per esempio, tra il 1387 e il 1410 si riscosse l'imposta tutti gli anni) ${ }^{19}$. Come abbiamo già detto, durante questo periodo, tutte le decime furono promulgate per soffocare le rivolte dell'isola di Sardegna pro recuperatione Sardinia et Corsica regni quod pro romana ecclesia in feudum tenor et etiam Camera Apostolica et romanam ecclesiam..$^{20}$

\section{I donativi generali e gli aiuti ex gratia}

Nonostante tutto, le entrate e la sicurezza che la decima forniva non erano sufficienti per il sovrano, il quale -come già detto- cercò sempre nuove vie per assicurarsi i redditi della Chiesa; ad esempio, nel 1371 il monarca aragonese scrisse a papa Gregorio XI, informandolo di aver già speso il denaro della decima concessagli (ancora prima di averla riscossa) per retribuire i soldati impegnati a domare le rivolte sarde. ${ }^{21}$ La situazione in cui si trovavano le casse regie e la necessità che le riscossioni fossero immediate, diedero luogo a contribuzioni parallele e, in un certo qual modo, legittime, benché, al momento della promulgazione della decima, esse avessero avuto una finalità diversa (guerra di Castiglia e difesa del territorio, matrimoni delle infanti, campagne in Sardegna, ecc.). Inoltre, i soggetti gravati dalle imposizioni potevano non essere esattamente gli stessi: infatti alla riscossione della decima e dei sussidi erano sottoposti gli ecclesiastici mentre alle tassazioni proclamate nelle Cortes e ad altri aiuti erano tenuti i sudditi laici che abitavano nelle signorie ecclesiastiche. Pertanto, possiamo dire che, oltre alla decima, esisteva anche una forma di contribuzione ecclesiastica negoziata. Questo era il caso dei sussidi e donativi concessi in maniera generale (nelle Cortes) o più particolare (nei Parlamenti) o degli aiuti specifici accordati al sovrano dagli ecclesiastici e dai loro vassalli.

19. Sull'alternanza delle decime per tutto il XIV secolo e per capire come la decima sia stata sostituita dai sussidi per gran parte del XV secolo:Morelló 2017; Tello 2019.

20. ACA, $R P, M R$ 1833, fol. 5r e ss. (anno 1375. Maiorca).

21. ASV, Reg. Aven. 174, fol. 434 (08/12/1317) (cf. LCGXI doc. 11948).
Così, da un lato, tanto in ogni singolo territorio come nell'insieme della Corona, i vari stamenti si riunivano in Cortes e Parlamenti durante i quali si concedevano donativi per i suddetti casi. Tali elargizioni dovevano riscuotersi mediante l'esazione delle imposte dirette e, soprattutto, indirette, che -in questo caso- interessavano abitualmente i vassalli delle signorie ecclesiastiche e, sempre a partire dalla seconda metà del XIV secolo, gli ecclesiastici stessi. Senza entrare nel merito della questione, il braccio ecclesiastico partecipò insieme agli altri ceti sociali in maniera continua alla maggior parte di queste assemblee generali fin dall'inizio della guerra contro la Castiglia, nel 1356 (non lo faceva dal 1282), e durante i decenni successivi, anche se opponendo, a volte, resistenza. ${ }^{22}$

Tuttavia, come abbiamo già detto, il clero tentava sempre di evitare una doppia contribuzione, ovvero di impegnarsi nel pagamento di sussidi di natura o finalità similari. Così, nelle Cortes aragonesi di Cariñena del 1357, gli ecclesiastici concessero un donativo rappresentato dalla fornitura di 200 uomini a cavallo. Nei capitoli di tale assegnazione, il braccio ecclesiastico chiese a Pietro IV il Cerimonioso di sospendere, nel frattempo, l'esazione della decima pontificia. Se, invece, questa fosse stata riscossa, i religiosi domandarono che almeno venisse successivamente decurtata dalla somma generale del donativo. ${ }^{23}$ A ogni modo, le suddette condizioni non sempre vennero accettate. A tale proposito, possiamo prendere come esempio quanto accadde nelle Cortes Generali di Monzón del 1362-1363. In questa assemblea generale, con rappresentanti di tutti gli stamenti e di tutti i territori della Corona, si concesse un donativo per i successivi due anni per far fronte alla guerra contro la Castiglia. ${ }^{24}$ Nella discussione con il braccio ecclesiastico, i chierici stabilirono che se il

22. Orti - Sánchez 1997, p. XVI-XXX. Come riferimento generale: Sánchez 1999a.

23. ACRA, t. II, p. 19, Item VII (11/08/1357). Nelle decime vigenti alcuni anni prima, già si esplicitava che non sarebbe stato permesso pretendere allo stesso tempo un altro sussidio o aiuto. Se ciò fosse accaduto, la decima doveva essere derogata: ordinamus quod si quid forsan a prelatis aut aliis personis ecclesiasticis regnorum, comitatuum, terrarum et dominiorum predictorum habuisti seu exegisti pro imminenti subsidio dicti regni Sardinie, id de hujusmodi concessa per nos tibi decima deducatur; ASV, Reg. Vat. 242, fol. 121 (28/02/1354) (cf. LSCIVI, doc. 793).

24. ACRA, t. II, p. 29-32, 271; Pons Guri 1982. 
papa avesse concesso una decima mentre si riscuoteva il donativo, la quantità con cui contribuivano gli ecclesiastici doveva essere detratta dalla decima. Il re non capiva questa richiesta di restituire parte di quanto riscosso perché riteneva che «no es justo quel senyor rey les de lo quel papa le da", cioè, il re non giudicava fosse giusto restituire agli ecclesiastici quello che il papa gli aveva concesso. Tuttavia, in un principio accettò che la decima di due anni, vigente in quel momento, si pagasse in quattro «e si mas les ne faze mas ${ }^{25}$.

Parallelamente alle Cortes e ai Parlamenti, il monarca sollecitò ulteriori aiuti particolari, sia perché quelli richiesti in occasione delle predette assemblee erano insufficienti, sia perché la loro riscossione era troppo lenta o anche per altre ragioni contingenti, come matrimoni delle infanti, coronazioni della regina o celebrazioni del regno. Le richieste alla Chiesa avevano sempre un carattere volontario; tuttavia, a partire dall'ultimo terzo del XIV secolo e nei primi decenni del XV esse divennero sempre più abituali. Tali disposizioni sono ben esemplificate dagli aiuti sollecitati per la celebrazione del giubileo del re. Nell'aprile del 1386, Pietro il Cerimonioso compiva 50 anni di regno, ragione per cui si preparò un grande giubileo e si fece richiesta di un importante sussidio in tutta la Corona così da poter affrontare le spese necessarie all'organizzazione dalla cerimonia (che poi, a quanto sembra, non si arrivò mai a festeggiare) ${ }^{26}$ La parte principale del sussidio per il giubileo è stato studiato da M. Sánchez alcuni anni fa e, per quanto riguarda la contribuzione ecclesiastica, abbiamo notizie circa le resistenze al pagamento opposte dai religiosi in tutta la Corona. Così, nella diocesi di Girona, Pietro IV incaricò due ufficiali della tesoreria perché tassassero e obbligassero gli ecclesiastici a pagare senza tener conto né di scuse, né di privilegi né di esenzioni anteriori. In particolare, il Cerimonioso si rivolse al vescovo Berengario di Anglesola, sollecitando il suo aiuto e avvertendolo che, nel caso in cui egli non avesse pagato ex gratia quanto dovuto, l'importo sarebbe stato calcolato dai commissari regi che avrebbero costretto gli ecclesiastici a pagare quanto loro

25. Orti-Sánchez 1997, p. 314. Comunque sia, non sappiamo con sicurezza se la riscossione della decima sia stata effettivamente prorogata.

26. Sánchez 1999b, p. 1453-1470. imposto, probabilmente prendendo a modello la decima pontificia. Infine, il sovrano alluse al fatto che tale contributo era necessario a causa delle grandi spese da affrontarsi per le celebrazioni del giubileo. ${ }^{27}$

La cessione di redditi ecclesiastici alla monarchia

Un'ulteriore forma di contribuzione ecclesiastica fu la cessione al re di redditi propriamente ecclesiastici in momenti particolari, quando l'esazione aveva finalità più $\mathrm{o}$ meno determinate. In concreto, ci riferiamo alle primicias e ai legati pii testamentari, impiegati, durante buona parte del periodo analizzato, per far fronte, principalmente, a spese relative alla difesa del territorio e alla manutenzione delle chiese. Per quel che ci interessa in questa sede, ci concentreremo sulle occasioni in cui questi redditi erano ceduti dal vescovo o dal papa alla monarchia. Le primicias costituivano -in teoria- una imposta percentuale gravante sulla produzione agricola e sull'allevamento che i laici consegnavano ai ministri della Chiesa. Durante la seconda metà del XIV secolo, i vescovi cedettero costantemente al re parte delle primicias, che finirono col trasformarsi in un tributo abituale di cui si beneficiarono i sovrani. I legati testamentari, d'altra parte, erano le disposizioni volontarie di beni o di quantità di denaro determinati nel testamento di un fedele che, in teoria, dovevano destinarsi a opere di carità. Anche questi redditi, puntualmente, furono utilizzati dal potere laico per la difesa della comunità, sempre con l'autorizzazione del vescovo o perfino del papa. ${ }^{28}$

In questo contesto, anche nell'ambito municipale si cercò di confiscare le primicias e i legati pii per far fronte a spese comunitarie e ad altre necessità urgenti, come, ad esempio, la costruzione di mura. Tale circostanza costituirà un ulteriore argomento utilizzato dal potere laico per giustificare la contribuzione forzata. A Barcellona, per esempio, si documentano assegnazioni di legati testamentari

27. Arnal 2000, doc. 351 (cf. Lletres reials de la ciutat de Girona, doc. 351. 04/05/1386). Così, il re ordinò a due funzionari regi di costringere al pagamento gli ecclesiastici della diocesi di Girona, senza tenere in conto i privilegi di esenzione da lui concessi in precedenza.

28. Tello 2017; Lafuente 2013, p. 221-226; Ledesma 1986, p. 61-63; ecc. 
per opere di fortificazione già a partire dalla fine del XIII secolo. ${ }^{29}$ Comunque sia, bisogna ricordare che, in ambito municipale, fu soprattutto durante la seconda metà del XIV secolo che si cercò di ottenere i legati pii e altri redditi per far fronte a tali tipi di esborsi. In questo senso, J. Vincke dimostrava come nel corso del settimo decennio del XIV secolo, le primicias furono impiegate nelle spese di costruzione o riparazione delle mura di città e cittadine, così come nella difesa delle frontiere, e arrivò un momento in cui il re le ricevette quasi in modo permanente. ${ }^{30}$ Tuttavia, nella documentazione conservata si dichiara costantemente che le entrate di quei redditi dovevano essere impiegate esclusivamente per la costruzione di mura e la riparazione di chiese, e mai per opere a carattere offensivo. ${ }^{31}$

\section{LA CONTRIBUZIONE FORZATA}

Oltre alle diverse forme tributarie esaminate finora, le autorità regia e municipale cercarono incessantemente di erodere anche l'esenzione degli ecclesiastici, violando o portando al limite la norma. Il risultato fu che questi comportamenti sfociarono nella riscossione di una vera e propria contribuzione forzata, ovvero in un'appropriazione indebita dei beni e dei redditi degli ecclesiastici. In questo senso, dobbiamo avvertire in anticipo che anche le autorità urbane tentarono di ricevere parte di questi redditi ecclesiastici, così troviamo qui un altro attore intorno alla pressione fiscale esercitata sugli ecclesiastici.

\section{L'erosione dell'immunità dei religiosi} ai confini del gruppo ecclesiastico

Lasciando da parte le discordie e gli scontri per la legittimità o meno dell'imposta e tenendo presente l'eterogeneità del gruppo ecclesiastico,

29. Verdés 2013, p. 114; Rovira i Solà 2008, doc. 185 (cf. Catàleg dels pergamins municipals de Barcelona, vol. I, doc. 185).

30. Vincke 1963, p. 276-277; Riu Riu 1996, p. 339.

31. Nelle Cortes aragonesi, il braccio ecclesiastico faceva notare ripetutamente che tale tassazione non doveva essere usata per fare la guerra o pagare i combattenti. Vedi, per esempio, i riferimenti delle Cortes di Cariñena del 1357 (ACRA, t. II, p. 191) o delle Cortes Generali di Monzón del 1362-1363 (ACRA, t.II, p. 314). dobbiamo fare riferimento a quei membri del clero che si situavano "a lato" della società laica. Li abbiamo definiti in questo modo perché tanto per il loro stile di vita quanto per la loro particolare condizione ecclesiastica, si collocavano ai limiti di questo gruppo eterogeneo. Così, all'interno della svariata gamma di religiosi, i tonsurati e gli ecclesiastici che praticavano il commercio si ponevano a cavallo tra il ceto ecclesiastico e il laicato. Tale condizione particolare stimolò la produzione di una legislazione ad hoc, volta a definire più precisamente gli obblighi contributivi di questi religiosi. Com'è noto, la tonsura indicava l'ingresso nell'ordine clericale. I tonsurati godevano dei privilegi del ceto ecclesiastico, erano soggetti alle leggi canoniche e a un "fuero" speciale, diverso da quello della società civile. Beneficiavano, dunque, del privilegium canonis e del privilegium fori. Tuttavia, il confine tra il mondo secolare e il mondo laico non era né chiaro né inamovibile. Difatti, gli stessi vescovi e i capitoli cattedrali erano coscienti di tali problemi, che causavano spesso abusi da parte dei tonsurati e il conseguente discredito dello stamento ecclesiastico. Già nel concilio di Saragozza del 1318 si dispose la realizzazione di un censimento che permettesse il controllo dei chierici tonsurati, regolamentando l'ingresso negli ordini sacri. ${ }^{32} \mathrm{E}$ ancora, in una prammatica del 1374, si ratificava una disposizione stando alla quale i chierici sposati, così come altri costituiti in ordini minori, tonsurati e accoliti che vantassero privilegi clericali, dovevano presentarsi al vescovo o all'ufficiale della loro diocesi nei tre mesi successivi alla loro ordinazione. In tal modo si cercò di controllare l'ordinazione ecclesiastica e, al tempo stesso, di ammonire coloro che erano tonsurati o portavano l'abito clericale senza, tuttavia, essere religiosi. ${ }^{33}$

Una seconda forma di limitazione dell'esenzione clericale è associata ai conflitti nel mondo ecclesiastico causati dall'espansione della fiscalità indiretta municipale durante il XIV secolo. Innumerevoli scontri seguirono all'obbligo dei religiosi ordinati di contribuire alla fiscalità indiretta, in particolare alle imposte (reali o municipali) che avevano un carattere indivisibile. Ci riferiamo, in questo caso, alle tasse che gravavano sul consumo

32. Aznar Gil 1982, p. 73.

33. ACA, Can. 1090, fol. 95v-96v (Barcelona. 06/03/1374); Rodrigo - Riera 2013, vol. 2, doc. 634. 
al dettaglio del pane, del vino o della carne, per le quali -in teoria- il clero doveva contribuire senza eccezione, a causa delle difficoltà che comportava distinguere tra il prezzo e l'imposta sul prodotto. ${ }^{34}$ Tuttavia, in alcuni casi, i religiosi avevano diritto alla restituzione di una somma di denaro equivalente all'imposta pagata. ${ }^{35}$ Così accadde intorno al 1371 in Catalogna: essendo stato gravato il clero con le imposte indirette sul commercio di panni e tessuti, una parte proporzionale di quanto riscosso nella decima pontificia del 1371 venne restituita agli ecclesiastici a ragione della "bolla dels draps». ${ }^{36}$

Per quanto riguarda l'imposta diretta possiamo osservare come numerosi municipi della Corona cercarono di limitare l'esenzione a quei religiosi che avevano ricevuto gli ordini maggiori e che avevano a disposizione beni strettamente ecclesiastici; in tal modo, si cercava di obbligarli a partecipare al pagamento delle taglie e delle imposte gravanti sui beni particolari di cui godevano. ${ }^{37}$ In merito a ciò, una forma di contribuzione documentata a margine dei tributi volontari fu il pagamento di tasse d'ammortizzazione per i beni che cadevano nella "mano morta», ossia quelle terre che, trasferite a ecclesiastici, sfuggivano alla tassa di trasferimento laica. Affinché i religiosi potessero continuare a disporre delle proprietà che avevano ricevuto dalla giurisdizione laica durante la loro vita, dovevano contribuire pagando delle licenze d'ammortizzazione. Queste tasse andavano a finire sia nelle casse regie, sia nell'erario municipale; infatti, è documentata una ricca produzione legislativa volta a regolare la

34. Ad esempio, questo è già stabilito in una lettera dei Consellers di Barcellona del 1346, dove si fa riferimento al fatto che gli ecclesiastici ordinati dovevano pagare solo alcuni tipi di tasse, mentre erano esentati dal pagamento delle imposizioni relative a "coses indivisibles, com son pa cuyt y carn...» (Rubriques de Bruniquer, vol. 2, cap. 27).

35. Le imposte su beni e consumi si espansero e si regolarizzarono nel corso del XIV secolo, diventando una delle principali fonti di reddito in molte città e cittadine della Corona. Come riferimento generale vedi Menjot - Sánchez 1999; Verdés 2004, p. 173-189.

36. Morelló 2013a, p. 495-504; 2013b, p.169-279, in particolare p. 192; 2013c.

37. Verdés 2013, p. 136-137; Sabaté 1998, p. 762-763. Nel caso di Perpignan, ad esempio, p. Verdés allude al fatto che disposizioni su tale materia esistevano già dal XIII secolo. Circa i tentativi dei pontefici di regolare l'intervento degli ecclesiastici nelle imposte indirette nel corso degli anni settanta del XIV secolo vedi ASV, Reg. Aven. 171, fol. 480v e ss $(15 / 08 / 1370)$. concessione di licenze come contropartita al diritto d'ammortizzazione. ${ }^{38}$

Infine, in relazione tanto alle imposte dirette quanto a quelle indirette, un argomento di contribuzione ricorrente e presente in tutti i territori europei furono le richieste per la fortificazione e la difesa delle città e delle cittadine a cui abbiamo già fatto riferimento. In questo caso, non stiamo più parlando di tassazione regie in senso lato, quanto piuttosto della tassazione laica imposta dai comuni e dalle città della Corona. Come dimostrato da numerosi studi sulle principali città europee, la costruzione di mura o di fortificazioni comportava necessariamente una contribuzione, in considerazione del fatto che tutti fruivano della protezione garantita dalle mura al nucleo urbano. ${ }^{39}$ Ancora una volta, constatiamo come i conflitti accesi dalle richieste di tasse per la costruzione di fortificazioni o per la difesa delle città aumentavano durante i periodi critici, come in occasione della guerra contro la Castiglia. Così, per esempio, intorno al 1369 scoppiò un famoso scontro tra il municipio di Valls (Tarragona) e il clero secolare per la contribuzione alle taglie. Nei decenni seguenti si conclusero vari patti e accordi che garantirono la contribuzione del clero, anche se con una serie di puntualizzazioni. ${ }^{40}$ Anche a Tarragona, negli anni sessanta del XIV secolo la contribuzione ai lavori per le mura causò un intenso conflitto. Oltre ai casi particolari, come ha dimostrato J. Morelló, i conflitti tra i sostenitori della giurisdizione ecclesiastica e di quella regia andarono aumentando durante gli anni della guerra contra la Castigla e nei decenni successivi, in un clima conflittuale che esulava dalla mera resistenza alla contribuzione fiscale. $^{41}$

38. Ferro 1987, p. 92; Palao 2001; Küchler 1997, p. 269-326. 39. Come riferimento generale, Rigaudière 2015; 1985, ecc.

40. Per l'ambito municipale, in generale, ci sono numerosi riferimenti in Verdés 2013, p.141-142; Turull 1997, p. 73-133. Sul conflitto di Valls: Morelló 1999, p. 697-708.

41. A questo proposito, si può vedere come sintesi generale il caso di studio di Valls: Morelló 2018, p. 177-210. Molti autori includono questi scontri nell'ambito dei cosiddetti "conflitti di temporalità", termine coniato da F. Sabaté per riferirsi ai contrasti avvenuti durante questi decenni tra il clero catalano e la monarchia: Sabaté 1998, p. 779-781; 2015, ecc. 
Le appropriazioni di redditi pontifici: l'esempio dello Scisma d'Occidente (13781387)

D'altra parte, come abbiamo già anticipato, la violazione diretta della norma è documentata anche dalle numerose testimonianze di appropriazione indebita di redditi ecclesiastici. Già negli anni cinquanta del XIV secolo, il cronista aragonese Jerónimo Zurita ci avverte della confisca di numerosi redditi ecclesiastici:

...essendo la sua necessità [del monarca] tanto grande che non era sufficiente con i suoi redditi e con i servizi che gli facevano, per mantenere la gente di guerra, ordinò di occupare i beni della camera apostolica e i frutti e i redditi di tutti i benefici dei cardinali e delle altre persone ecclesiastiche che si trovavano assenti dai loro benefici. E questo si fece con editti pubblici. ${ }^{42}$

Allo stesso modo, nel contesto della guerra contra la Castiglia (1356-1366), è attestato che, per un breve periodo, Pietro IV cercò di usurpare i redditi dei chierici assenti dai benefici, sempre in un contesto di appropriazione di beni e rendite appartenenti a Castigliani e Navarri in territori della Corona. ${ }^{43}$

Comunque sia, il numero delle richieste generali andò aumentando nel corso degli anni settanta del XIV secolo, quando, senza contare le decime che si susseguirono tra il 1372 e il 1379 , il braccio ecclesiastico contribuì a numerosi donativi disposti in diverse Cortes per finanziare la difesa del territorio e per soffocare le rivolte della Sardegna, così come a diversi sussidi sollecitati dal monarca per i matrimoni delle infanti e dell'infante. Difatti, tali

42. Anales, vol. IV, 1. IX, cap. 53. Ovviamente, il papato richiese il rispetto delle esenzioni garantite al clero e la propria approvazione per qualunque tipo di contribuzione ecclesiastica. Vedi un riferimento, ad esempio, in ASV, Reg. Vat. 236, fol. 43r (17/03/1354).

43. Questa realtà fa parte di un contesto piuttosto concreto dove scopriamo che l'interesse della monarchia era quello di appropriarsi dei redditi del clero assenteista e limitare l'accesso ai benefici agli ecclesiastici stranieri, alludendo al fatto che essi non risiedevano in loco e sfuggivano, quindi, al controllo regio. Entrambe le questioni non hanno goduto di studi nella Corona di Aragona nonostante siano perfettamente inquadrabili in un contesto comune ad altri territori europei: Vincke 1963, p. 272. spese incidevano enormemente sull'indebitamento della monarchia. In questo contesto, la sempre maggiore pressione fiscale causò quel particolare fenomeno rappresentato dall'appropriazione di redditi della Camera Apostolica. Com'è noto, nel 1378 ebbe inizio lo Scisma d'Occidente, momento a partire dal quale convissero due pontefici; uno a Roma e un altro ad Avignone. Da allora, le monarchie cristiane si schierarono ora con uno ora con l'altro papa, a seconda delle alleanze e degli equilibri tra le forze in campo. ${ }^{44}$ Tuttavia, nella Corona d'Aragona, il re Pietro IV si dichiarò indifferente o neutrale e non riconobbe nessuno dei due pontefici. Il risultato fu che, dal 1379 al 1387, il monarca si appropriò delle diverse esazioni che avevano come destinazione ordinaria la Camera Apostolica di Avignone, rimanendo il loro prodotto in potere della Corona. Per questo motivo, vale la pena approfondire alcune di esse, come l'annata o i servitia communia, che venivano pagati al momento dell'assegnazione di un nuovo beneficio o di uno rimasto vacante, così come anche lo ius spoli. Benché fossero redditi straordinari e sporadici, in questo periodo erano spesso reclamati. Nello specifico, nella Corona d'Aragona quando un beneficio rimaneva vacante i suoi profitti erano destinati alla Camera Regia. ${ }^{45}$

Per gestire questi redditi si creò un corpo amministrativo ad hoc, che la storiografia definì, molti anni fa, come Cámara Apostólica Real. Dalla monarchia venne nominata una persona vicina alla cancelleria regia in qualità di ricevitore generale e distributore dei redditi della Camera Apostolica nella Corona d'Aragona. Inoltre, venne designato un numero variabile di commissari generali incaricati della riscossione di redditi e questi, a loro volta, nominarono altri commissari particolari o locali che operavano nelle varie circoscrizioni della Corona. Per l'oggetto precipuo della presente ricerca bisogna sottolineare come, nei diplomi con i quali il sovrano incaricava tali commissari, si faceva spesso riferimento all'ordine regio di requi-

44. Ad esempio, Castiglia, Francia e Napoli obbedirono al papa di Avignone Clemente VII, mentre l'Inghilterra era fedele al papa romano Urbano. A questo proposito, A. Rehberg mostra come tra il 1378-1381 i regni iberici portarono avanti numerose consultazioni realizzando vari incontri con le élite religiose e politiche per decidere l'obbedienza all'uno o all'altro Papa: Rehberg 2009, p. 247-305.

45. Tello 2016, p. 201-222; Ivars 1928. 
sizione dei redditi della Camera fino al momento in cui fosse stato eletto il papa legittimo. Solo allora i redditi sarebbero stati restituiti, il che obbligava il re ad adottare un atteggiamento neutrale e «a esser indiferent a obehir a algú dels dits elets, tro que fos declarat qual d'aquelles obtendria lo papat». ${ }^{46}$ Tuttavia, questi redditi furono impiegati per far fronte alle imprese più urgenti del monarca in quel decennio: sedare le rivolte della Sardegna, appianare i numerosi prestiti del monarca e sanare le esauste finanze regie. ${ }^{47}$

\section{CONCLUSIONE}

Da quanto esposto, possiamo concludere che nel momento in cui richiedeva la contribuzione degli ecclesiastici, il potere civile adottò tre modelli di comportamento fondamentali. In primo luogo, il monarca e i municipi fecero il possibile per far sì che lo stamento ecclesiastico contribuisse in maniera volontaria alle richieste, così come stabilivano la legislazione canonica e civile. Quindi, si cercò di fare in modo che i chierici partecipassero al pagamento delle imposte allo stesso modo dei laici nei casi in cui il confine giurisdizionale tra i due stamenti non era chiaro, tanto per la condizione giuridica del singolo ecclesiastico e dei suoi beni, quanto per la natura e la finalità dell'aiuto sollecitato. Infine, come detto in precedenza, in particolari momenti si optò anche per l'usurpazione di redditi e beni ecclesiastici, violando in modo più o meno giustificato la normativa vigente.

In generale, dobbiamo tenere presente come, dalla metà del XIII secolo e per tutto il XIV, si verificò un profondo cambiamento nelle strutture fiscali e finanziarie dell'Occidente medievale. I sovrani cercarono nuove risorse per rimpinguare le casse reali e uno dei modi per ottenerle fu richiedere un aiuto da parte del clero. A tal fine, sono stati articolati discorsi elaborati che ruotavano intorno alla giustificazione della tassa e al contributo degli ecclesiastici, così come abbiamo visto. ${ }^{48}$ In ogni caso, a seguito di questa strategia,

46. ACA, RP, $M R, 650$, fols. 34v-37v (Barcelona. 26/09/1380). 47. Tello 2016, p. 209-213.

48. Genet - Le Page - Mattéoni 2017; Hèbert 2017, ecc. Sui rapporti tra il papato e la monarchia d'Aragona in questi decenni, con particolare riferimento alle questioni di natura fiscale, vedi Sabanés - Schmidt 2016; Vincke 1936. nel corso del XIV secolo, si può osservare come la monarchia aragonese si presentasse nelle vesti della legittima e abituale beneficiaria di certi tributi o redditi ceduti dal Papa (nel caso della decima) o dalla corrispondente autorità ecclesiastica (nel caso delle primicias o causas pías).

Oltre a ciò, la definizione del gruppo ecclesiastico in relazione alla norma giuridica e alla pratica fiscale rende evidente come non tutti i chierici erano uguali, né riguardo all'ordine sacerdotale ricevuto, né riguardo alla giurisdizione ecclesiastica goduta; esisteva una frontiera permeabile nella quale si attestano esempi della eterogeneità del gruppo sociale e delle sue diverse forme di contribuzione all'erario regio e municipale. Inoltre, malgrado qui se ne sia stato fatto solo un breve accenno, è necessario segnalare anche l'esistenza di una problematica relativa alla contribuzione indiretta a cui erano sottoposti i vassalli della Chiesa. Non è sempre facile accertare se tale tassazione ricadesse sui chierici in generale, sui signori ecclesiastici, sui loro vassalli o sui contribuenti della fiscalità ecclesiastica. In altre parole, è necessario distinguere le rendite che derivavano dallo stato di subordinazione degli uomini soggetti al braccio ecclesiastico in ragione della loro giurisdizione temporale, da quelle connesse alla giurisdizione spirituale, che si concretizzavano nel contributo ecclesiastico vero e proprio. È su quest'ultima forma in particolare, che ci siamo concentrati nella presente indagine.

Per concludere, abbiamo tracciato dei brevi appunti su come, malgrado la sua teorica esenzione, il clero della Corona d'Aragona del basso Medioevo finì col contribuire in maniera effettiva e crescente alle diverse richieste fiscali avanzate dal potere laico. Oltre l'ambito fiscale, tutto ciò comportò una resistenza da parte del ceto ecclesiastico di fronte al potere laico, e allo stesso tempo si crearono interessanti legami e forme di negoziazione che esemplificano perfettamente le sinergie e le relazioni tra poteri durante il basso Medioevo. In questo modo, osserviamo, quindi, il ruolo di primo piano assunto dalla Chiesa, che venne a crearsi soprattutto durante il XIV secolo, quando la Corona infranse tutti i limiti tradizionali relativi alla contribuzione ecclesiastica e andò via via sempre più erodendo la teorica esenzione del clero per alleviare la critica situazione delle finanze reali. Così, come abbiamo visto, non erano più sufficienti le decime cedute al monarca dal papato e neanche i donativi ex gratia elargiti nelle Cortes; 
si pretendeva che anche gli ecclesiastici e i loro vassalli contribuissero alle richieste straordinarie sollecitate dalla monarchia in diverse circostanze. Tutto ciò comportava entrate rilevanti per il fisco regio, a cui i monarchi non erano disposti a rinunciare. Infine, oggi non siamo in grado di dare una risposta precisa alla questione relativa alla consistenza effettiva del contributo ecclesiastico raccolto in tutta la Corona, ma sicuramente è possibile approfondire la tematica grazie alla preziosa docu-

\section{Bibliografia}

\section{Archivi}

ASV $=$ Archivio Segreto Vaticano.

ACA = Archivio della Corona d'Aragona.

\section{Bibliografia primaria}

ACRA = Acta Curiarum Regni Aragonum. Tomo II Cortes del reinado de Pedro IV/1. ed. a cura di J.Á. Sesma Muñoz, M. Lafuente Gómez, Saragozza, Gobierno de AragónIbercaja, 2013.

Anales = Anales de Aragón de Jerónimo Zurita (1512-1580), ed. A. Canellas López, Saragozza, Institución Fernando el Católico, 1967 (edición electrónica 2003).

Arnal 2000 = J. Arnal, Lletres reials de la ciutat de Girona (1293-1515), Barcellona, Fundació Noguera, 2000.

Rodrigo - Riera 2013 = M. Rodrigo, J. Riera, Col-lecció documental de la Cancelleria de la Corona d'Aragó: Textos en llengua catalana (1291-1420), Valencia, Publicacions de la Universitat de València, 2013.

Rovira i Solà $2008=$ M. Rovira i Solà, Catàleg dels pergamins municipals de Barcelona (volums II y III), Barcellona, Ajuntament de Barcelona, 2008.

LCGXI = A.M. Hayez et alii (a cura di), Grégoire XI (13701378). Lettres communes analysées d'après les registres dits d'Avignon et $d u$ Vatican, vol. I-III, Rome, École française de Rome, 1990-1998.

LSCIVI = M.H. Laurent, N. Gotteri (a cura di), Innocent VI (1352-1362). Lettres secrètes et curiales, Roma, École française de Rome, 1959-2006.

Orti - Sánchez 1997 = M. Sánchez, P. Orti (a cura di), Corts, parlaments i fiscalitat a Catalunya. Els capitols del donatiu (1288-1384), Barcellona, Textos jurídics catalans - Generalitat de Catalunya, 2007.

Pons Guri 1982 = J. M. Pons Guri (a cura di), Actas de mentazione ancora oggi conservata. In ogni caso, possiamo affermare che il contributo ecclesiastico ha avuto un peso crescente sulle finanze regie durante i secoli XIV-XV. Le risorse della Chiesa non erano controllate direttamente né dalle Cortes né dalla Diputación del General, e il monarca poteva disporne in modo più o meno diretto, a seconda della bontà dei rapporti con la curia pontificia. ${ }^{4}$

las Cortes Generales de la Corona de Aragón de 1362-1363, Madrid, Subdirección General de Archivos, 1982.

Rubriques de Bruniquer $=\mathrm{G}$. Esteve Bruniquer, Rúbriques. Ceremonial dels magnifichs Consellers y Regiment de la Ciutat de Barcelona, Barcellona, Impr. D'Henrich, 5 vols., 1912-1916.

Sabanés - Schmidt 2016 = R. Sabanés, T. Schmidt (eds.), Butllari de Catalunya: documents pontificis originals conservats als arxius de Catalunya (1198-1417), Barcellona, Fundació Noguera, 4 vols., 2016.

\section{Bibliografia secondaria}

Aznar Gil 1982 = F. Aznar Gil, Concilios provinciales y sinodos de Zaragoza de 1215 a 1563, Saragozza, 1982.

Barralis - Genet $2014=$ J.-Ph. Barralis, J.-Ph Genet et al (a cura di), Église et État, Église ou État? Les clercs et la genèse de l'État moderne, Parigi, 2014.

Baucells 2004-2007 = J. Baucells, Vivir en la Edad Media: Barcelona y su entorno en los siglos XIII y XIV (1200-1344), Barcellona, vol. III, 2004-2007, p. 2277-2292.

Bertran $1997=$ P. Bertran, La fiscalitat eclesiàstica en els bibast catalans (1384-1392): Tipologies impositives y diferències territorials, in Acta historica et archaeologica mediaevalia, 18, 1997, p. 281-300.

Bertran $1990=$ P. Bertran, El poder de l'Església medieval: organització administrativa $i$ sistema fiscal en el segle XIV, in L'Avenç, 139, 1990, p. 66-69.

49. Per approfondire tale argomento vedi Morellò 2017. Relativamente al contesto del XV secolo si può far riferimento a Küchler 1996. 
Bonney 1995 = R. Bonney, Economic systems and state finance, Oxford, 1995.

Bonney $1999=\mathrm{R}$. Bonney, The rise of the fiscal state in Europe c. 1200-1815, Oxford, 1999.

Carretero 2013 = J.M. Carretero, La Colectoría de España en el siglo XVI: los mecanismos de transferencia monetaria entre España y Roma (cambios y créditos), in Hispania. Revista española de historia, 73, 2013, p. 79-104

Cevins - Matz 2010 = M.M. Cevins, J.M. Matz (a cura di), Structures et dynamiques religieuses dans les sociétés de l'Occident latin (1179-1449), Rennes, 2010.

Davis $2014=\mathrm{V}$. Davis, Clerics and the king's service in late medieval England, in Barralis - Genet 2014, p. 25-34.

Favier $1966=\mathrm{J}$. Favier, Les finances pontificales à l'époque du gran schisme d'Occident (1378-1409), Parigi, 1966.

Ferro $1987=$ V. Ferro, El dret Públic Català Les Institucions a Catalunya fins al Decret de Nova Planta, Vic, 1987.

Fortea 2015 = J. I Fortea, La Hacienda de los Estados Pontificios en los inicios de la Modernidad (1420-1565), in M.Á. Ladero et al (a cura di), Estados y mercados financieros en el Occidente cristiano (siglos XIII-XVI). Actas de la XLI Semana de Estudios Medievales de Estella (2014), Pamplona, 2015, p. 473-508.

Garnier 2013 = F. Garnier, Justifier le financement de la dépense au Moyen Âge, in A. Galán, J.M. Carretero (a cura di), El alimento del Estado y la salud de la res publica: Orígenes, estructura y desarrollo del gasto público en Europa, Madrid, 2013, p. 51-73.

Genet - Le Page - Mattéoni 2017 = J.P. Genet, D. Le Page, O. Mattéoni (a cura di), Consensus et représentation : actes du colloque organisé en 2013 à Dijon, Paris, 2017.

Genet - Vicent 1986 = J.Ph Genet, B. Vicent (a cura di), État et Église dans la genèse de l'État moderne, Madrid, 1986.

Giannini 2014 = M.C. Giannini (a cura di), Fiscalità e religione nell'Europa cattolica, Roma, 2014.

Hébert $2017=$ M. Hébert Consensus et représentation en Europe occidentale, XIII ${ }^{e}$ XVII siècle. Une introduction, in J.P. Genet, D. Le Page, O. Mattéoni (a cura di), Consensus et représentation : actes du colloque organisé en 2013 à Dijon, Parigi, 2017, p. 11-40

Housley $1986=\mathrm{N}$. Housley, The Avignon papacy and the crusades 1305-1378, Oxford, 1986.

Isenmann $2010=\mathrm{E}$. Isenmann, The notion of the common good, the concept of politics, and practical policies in late medieval and early modern German cities, in Lecuppre - Van Bruaene 2010, p. 107-148.

Ivars $1928=\mathrm{A}$. Ivars, La «indiferencia» de Pedro IV de Aragón en el gran cisma de occidente: 1378-1382, Madrid, 1928.

Lecuppre - Van Bruaene 2010 = É. Lecuppre, A.L. Van Bruaene (a cura di), De bono communi. The discourse and practice of the common good in the European City, Turnhout, 2010, p. 107-148.

Jamme 2005 = A. Jamme, Renverser le pape. Droits, complots et conceptions politiques aux origines du Grand Schisme d'Occident, in F. Foronda, J.Ph. Genet et al. (a cura di), Coups d'État à la fin du Moyen Âge? Aux fondements du pouvoir politique en Europe occidentale, Madrid, 2005, p. $433-482$

Jamme 2015 = A Jamme, Le pape et les princes. Deux cas d'espèces: Clericis laicos, in A. Berenguer, O. Dard (a cura di), Gouverner par les lettres, de l'antiquité à l'epoque contemporaine, Loraine, 2015, p. 117-146.

Kempshall $1999=$ M.S. Kempshall, The common good in late medieval political thought, Oxford, 1999.

Küchler 1996 = W. Küchler, Les finances de la Corona d'Aragó al segle $X V$ (Regnats d'Alfons $V i$ Joan II), Valencia, 1997.

Ladero Quesada et al. 2015 = M.A. Ladero Quesada et al. (a cura di), Estados y mercados financieros en el Occidente cristiano (siglos XIII-XVI). Actas de la XLI Semana de Estudios Medievales de Estella (2014), Pamplona, 2015.

Lafuente $2013=$ M. Lafuente, Un reino en armas. La guerra de los Dos Pedros en Aragón (1356-1366), Saragozza, 2013.

Ledesma 1986 = M.L. Ledesma, El uso de las primicias eclesiásticas por los monarcas aragoneses en la segunda mitad del siglo XI, in Simposio Nacional sobre ciudades episcopales, Saragozza, 1986, p. 61-67.

Lecuppre 2010 = E. Lecuppre et al (a cura di), De bonno communi. The discourse and practice of the common good in the European city, Turnhout, 2010.

Lunt 1943 = W.E. Lunt, Papal revenues in the Middle Ages, New York, 1943 (reimp. 1965).

Menjot - Sánchez 1996 = D. Menjot, M. Sánchez (a cura di), La fiscalité des villes au Moyen Âge (France méridionale, Catalogne et Castille). Étude des sources, Tolosa, 1996.

Menjot - Sánchez 2011 = D. Menjot, M Sánchez (a cura di), El dinero de Dios. Iglesia y fiscalidad en el Occidente Medieval (siglos XIII-XV), Madrid, 2011.

Menjot - Sánchez 1999 = D. Menjot, M. Sánchez (a cura di), La fiscalité des villes au Moyen Âge (Occident méditerraneen). 2. Les systèmes fiscaux, Tolosa, 1999.

Millet $2010=\mathrm{H}$. Millet, Qu'est-ce que la papauté avignonnaise?, in Lusitania Sacra, 22, 2010, p. 17-25.

Millet $2013=\mathrm{H}$. Milet, L'Église du Grand Schisme. 13781417, Paris, 2013.

Moeglin - Péquignot 2017 = J.-M. Moeglin, S. Péquignot (a cura di), Diplomatie et "relations internationales» au Moyen Âge (IXe-XVe siècle), Parigi, 2017.

Montagut $2004=\mathrm{T}$. de Montagut, El sistema del derecho tributario común en la Corona de Aragón: Notas para su estudio, in O. Condorelli (a cura di), "Panta rei»: Studi dedicati a Manlio Bellomo, Roma, 2004, p. 49-68.

Morelló 1999 = J. Morelló, "De contributionibus fiscalibus": els conflictes entre el municipi de Valls i la comunitat de preveres durant el segle XIV, in Anuario de Estudios Medievales, 29, 1999, p. 689-729.

Morelló 2009 = J. Morelló, La maquinaría fiscal del papado aviñonés en la Corona de Aragón: la colectoría de Berenguer Ribalta (1400-1402), in Anuario de Estudios Medievales, 39-1, 2009, p. 65-125.

Morelló 2013a = J. Morelló (a cura di), Financiar el reino terrenal: la contribución de la Iglesia a finales de la Edad Media (siglos XIII-XVI), Barcellona, 2013.

Morelló $2013 \mathrm{~b}=\mathrm{J}$. Morelló, El clero de Catalunya davant els impostos del general: a propòsit de la compravenda de draps al bisbat de Barcelona a la dècada de 1370. Morelló 2013a, p. 169-279.

Morelló 2013c = J. Morelló, El clero de Catalunya davant 
els impostos del General: La polèmica sorgida a la dècada de 1370 al voltant de la doble contribució eclesiàstica, in J. Mutgé, R. Salicrú, et al. (a cura di), La Corona catalanoaragonesa, l'islam i el món mediterrani, Barcellona, 2013, p. 495-504.

Morelló 2017 = J. Morelló, En torno a la disyuntiva décimal subsidio en Castilla y la Corona de Aragón durante la Baja Edad Media, in Hispania, 257, 2017, p. 643-671

Morelló 2018 = J. Morelló, En defensa de la libertad eclesiástica. Actuación corporativa del clero de la Tarraconense $y$ establecimiento de tallas conciliares en los años que precedieron a la concordia de 1372, in J. Morelló, P. Orti, P. Verdés (a cura di), Renda feudal $i$ fiscalitat a la Catalunya Baixmedieval. Estudis dedicats a Manuel Sánchez Martínez, Barcellona,2018, p. 177-210.

Ormrod - Killick - Bardford 2017 = W.M. Ormrod, H. Killick, P. Bradfort (a cura di), Early common petitions in the English Parliament, c. 1290-1420, Cambridge, 2017.

Ortego $2009=$ P. Ortego, Justificaciones doctrinales de la soberanía fiscal regia en la baja Edad Media castellana, in En la España Medieval, 32, 2009, p. 113-138.

Ortego 2012 = P. Ortego, Las riquezas de la Iglesia al servicio del poder monárquico: los empréstitos eclesiásticos en la Castilla del siglo XV, in La España Medieval, 35, 2012, p. 145-176.

Orti - Sánchez 1997 = P. Orti, M. Sánchez, La Corona en la génesis del sistema fiscal municipal en Cataluña (13001360), in M. Sánchez, P. Bertran, A. Furio (a cura di), Colloqui Corona, Municipis i Fiscalitat a la Baixa Edat Mitjana, Leida, 1997, p. 233- 278.

Palao 2001 = F.J. Palao Gil, La propiedad eclesiástica y el juzgado de amortización de Valencia (siglos XIV-XIX), Valencia, 2001.

Payan 2010 = P. Payan, Entre Rome et Avignon. Une histoire du Gran Schisme (1378-1417), Parigi, 2009.

Rehberg 2009 = A. Rehberg, Le inchieste dei re d'Aragona e di Castiglia sulla validità dell'elezione di Urbano VI nei primi anni del Grande Scisma. Alcune piste di ricerca, in L'età dei processi. Inchieste e condanne tra politica e ideologia nel'300. Atti del convegno (Ascoli, 2007), Roma, 2009, p. 247-305.

Rigaudière 1985 = A. Rigaudiere, Le financement des fortifi cations urbaines en France du milieu du XIV siècle à la fin du XV siècle, in Revue Historique, 273, 1985, p. 19-95.

Rigaudière 2015 = A. Rigaudière, Le clerc, la ville et l'impôt dans la France du Bas Moyen Âge, in M.C. Giannini (a cura di), Fiscalità e religione nell'Europa cattolica, Roma, 2014, p. 22-23.

Riu Riu 1996 = M. Riu Riu, El Poder Real y la Iglesia catalana en la Corona de Aragón (siglos XIV al XVI), in El Poder Real en la Corona de Aragón (siglos XIV-XV). Actas del XV Congreso de Historia de la Corona de Aragón (Jaca, 1993), vol. I, Saragozza, 1996, p. 389-407.

Sabaté $1995=$ F. Sabaté, Discurs i estratègies del poder reial a Catalunya al segle XIV, in Anuario de Estudios Medievales, 25-2, 1995, p. 617-546.

Sabaté 1998 = F. Sabaté, L'Església secular catalana al segle XIV: la conflictiva relació social, in Anuario de Estudios Medievales, 28, 1998, p. 757-788.
Sánchez 1999b = M. Sánchez Martínez, La última ofensiva fiscal de Pedro el Ceremonioso: las demandas para el jubileo de 1386, in Aragón en la Edad Media, 14-15, 1999, p. $1453-1410$.

Sánchez 2009a = M. Sánchez Martínez, Cortes y fiscalidad: el caso de Cataluña durante la segunda mitad del siglo XIV, in Aragón en la Edad Media, 21, 2009, p. 279-308.

Sánchez 2011 = M. Sánchez Martínez, La participación de la Iglesia de Cataluña en las finanzas regias (siglos XIII$X I V)$, in D. Menjot, M Sánchez (a cura di), El dinero de Dios. Iglesia y fiscalidad en el Occidente Medieval (siglos XIII-XV), Madrid, 2011, p. 133-167.

Sánchez - Furio - Sesma 2008 = M. Sánchez, A. Furio, A. Sesma, Old and new forms of taxation in the Crown of Aragon, in La fiscalità nell'economia europea (sec. XIIIXVIII). 39 Settimana di Studi dell'Istituto internazionale di storia economica "Francesco Datini" di Prato, Firenze, 2008, p. 99-130.

Scordia 2005 = L. Scordia, Le roi doit vivre du sien. La théorie de l'impôt en France (XIII'-XVe siècles), Paris, 2005.

Souza - Bayona 2014 = J.A. Souza, B. Bayona (a cura di), Doctrinas y relaciones de poder en el Cisma de Occidente y en la época conciliar (1378-1449), Saragozza, 2014.

Tello 2016 = E. Tello Hernández, La Cámara Apostólica Real en la Corona de Aragón: aproximación a su estructura institucional y aparato financiero (1378- 1387), in P. Iradiel, G. Navarro, D. Igual, C. Villanueva (a cura di), Identidades urbanas Corona de Aragón-Italia. Redes económicas, estructuras institucionales, funciones politicas (siglos XIV-XV), Saragozza, 2016, p. $201-222$.

Tello 2017 = E. Tello Hernández, Pro defensione regni: la contribución de la iglesia a las demandas de pedro iv de Aragón (1349-1387), Tesis inédita de la Universidad de Zaragoza, Saragozza, 2017.

Tello 2019 = E. Tello Hernández, La comptabilité des décimes pontificales dans la Couronne d'Aragon (1309-1456), in Comptabilités. Revue d'histoire des comptabilités, 10, 2019, http://journals.openedition.org/comptabilites/2491.

Turull $1997=$ M. Turull Rubinat, El impuesto directo en los municipios catalanes medievales, in Finanzas y fiscalidad municipal, Leon, 1997, p. 73-133

Vincke 1936 = J. Vincke, Documenta selecta mutuas civitatis Arago-Cathalaunicae et ecclesiae relationes illustrantia, Barcellona, 1936.

Verdés $2004=\mathrm{P}$. Verdés, La gestión de los impuestos indirectos municipales en las ciudades y villas de Cataluña: el caso de Cervera (s. XIV-XV), in D. Menjor, M. Sánchez (a cura di), La fiscalité des villes au Moyen Âge (Occident méditerranéen). La gestion de l'impôt, Tolosa, 2004, p. 173-189.

Verdés $2013=$ P. Verdés, La contribución eclesiástica a la fiscalidad municipal en Cataluña durante la época bajomedieval, in Morelló 2013a, p. 131-168.

Vincke 1963 = J. Vincke, Estado e Iglesia en la Historia de la Corona de Aragón de los siglos XII, XII y XIV, in Jerónimo Zurita. La seva obra i l'estat general de la investigació històrica. Actas del VII Congreso de Historia de la Corona de Aragón (Barcelona, 1962), vol. I, Barcellona, 1963, p. 267-285. 


\title{
Table des Matières
}

\author{
Premier fascicule
}

\section{BIBLIOTHÉRAPIES MÉDIÉVALES}

sous la direction de Florent Coste

Bibliothérapies médiévales. Pour une généalogie des pouvoirs curatifs du livre, par Florent Coste. . . . . . . . . .

Pourquoy li aucun dorment qui se prendent a lire» : représentations médiévales des effets soporifiques et des vertus

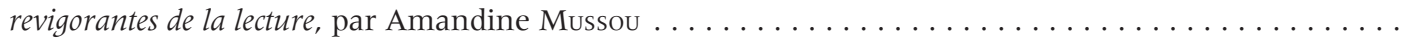

La parola terapeutica del papa: prime indagini sui sermoni di Onorio III, par Christian GRAsso. . . . . . . . . . .

Stimuli, Remedia e altre immagini: sulla rivoluzione devozionale dei francescani, par Antonio MoNTEFusCo. .

L'Inflammatorium poenitentiae ou comment lutter contre l'acédie, par Florent Coste . . . . . . . . . . .

L'écriture dans l'image médiévale. Espaces libérés du sens et de l'imagination, par Vincent DeBIAIs. . . . . . . . .

\section{VARIA}

Un libellus poetico all'origine dei Carmina Ratisponensia? Una nuova proposta sulla genesi della raccolta, par

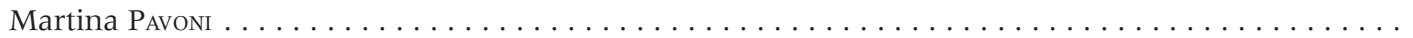

Le entrate e le risorse materiali dei conti Guidi negli anni '20 del secolo XIII: una stima complessiva, par Tommaso

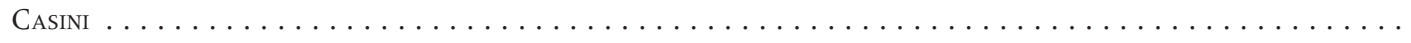

Les mots et les images de l'adoration. La Nativité dans les Méditations de la vie du Christ (Italie, début

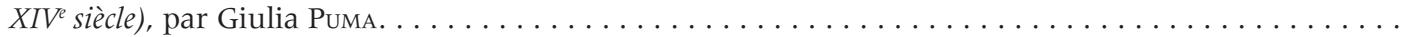

Rappresentazioni del potere tra giudicato d'Arborea, Corona d'Aragona e Regno di Napoli, par Nicoletta UsAI .

Res publica restituta? Perceiving Emperors in Fourteenth-Century Rome, par Anne HuIJbers . . . . . . . . . . .

Taormina 1465: la concessione del teatro antico (lu Goliseu alias lu Palazu) come residenza signorile e altri casi di riuso di monumenti antichi nella Sicilia del XV secolo, par Francesco Muscolino . . . . . . . . . . . . . . . .

\section{ATELIER DOCTORAL: OUTILS ET MÉTHODES POUR L'HISTOIRE DES ÉGLISES ENTRE ORIENT ET OCCIDENT}

sous la direction Camille Rouxpetel et Frédéric GabRieL

Introduction, par Camille Rouxpetel et Frédéric Gabriel $\ldots \ldots \ldots \ldots \ldots \ldots \ldots \ldots \ldots \ldots \ldots$

Il De episcoporum transmigratione, le decretali pseudo-isidoriane e $i$ dibattiti sul trasferimento e la deposizione dei vescovi tra la metà del IX e l'inizio del X secolo, par Giulia Cò . . . . . . . . . . . . . . . . . . .

The content and the ideological construction of the early pontifical manuscripts, par Arthur WeSTWELL .......

Le rôle de tribus arabes chrétiennes dans l'intégration de l'Orient à l'Église syro-orthodoxe : de la mort de Sévère (v. 683-684) à la crise entre Denhạa II et Julien II (r. 687-709), par Simon PIERre . . . . . . . . . . . . . . . . . . .

Entre la tiare et le turban. le clerge grec orthodoxe face a la domination ottomane (XVI'-XVII siecles), par Alexandra

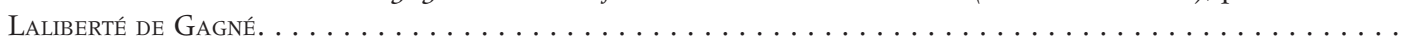




\section{Deuxième fascicule}

PERSONE, CORPI E ANIME IN MOVIMENTO. FORME DI MOBILITÀ TRA TARDOANTICO E ALTO MEDIOEVO (VI-X SECOLO)

sous la direction de Annamaria Pazienza et Francesco Veronese

Introduzione, par Annamaria PAzIENZA et Francesco Veronese .

Collocare le anime nell'aldilà: rappresentazioni dell'inferno nei Dialogi di Gregorio Magno, par Denise DeIANA.

Mobilité des corps et transfert des os: le remploi des sépultures dans cinq nécropoles mérovingiennes

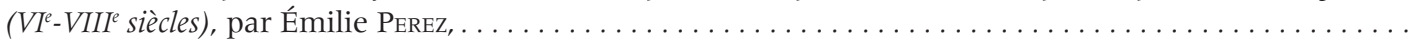

Mobilità e controllo politico nel regno longobardo: fonti normative e riflessi documentari, par Gianmarco DE

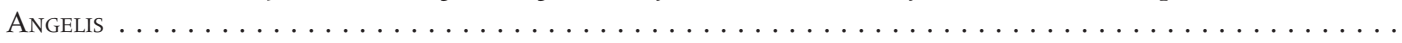

«Cum p(re)misso Masarię socrus meę». Mobilità residenziale e sposi migranti nel regno longobardo, par

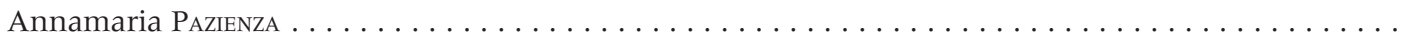

Tra rispetto delle leggi e furti notturni. Narrazioni carolinge di spostamenti di reliquie, par Francesco VERONESE On moving bodies and monastic history. The relics and Vita of Probus of Ravenna, par Edward Schoolman ... Conclusioni, par Annamaria PazIenza e Anna RAPETti

\section{VARIA}

La Sardegna nel Mediterraneo di VII-VIII secolo attraverso il dato archeologico, numismatico e sfragistico, par

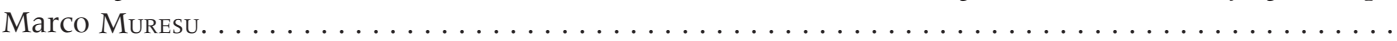

Uso politico delle reliquie e modelli di regalità longobarda da Liutprando a Sicone di Benevento, par Alessandro

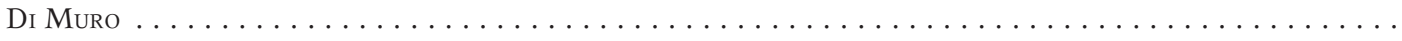

La messa in scena iconica del corpo regio nel regno di Sicilia (1130-1266).

Alessandra Acconci, Frammenti di un ciclo dei Mesi a Ceccano, par Mirko Vagnoni . . . . . . . . . . . . . .

Nichil solvit. Norma e pratica nella contribuzione ecclesiastica per la monarchia nella Corona d'Aragona durante il papato di Avignone e lo Scisma (1309-1418), par Esther Tello Hernández. . . . . . . . . . . . . . . . . . . . Quadri prosopografici della feudalità calabrese in età angioina: i Ruffo di Calabria tra XIV e XV secolo, par

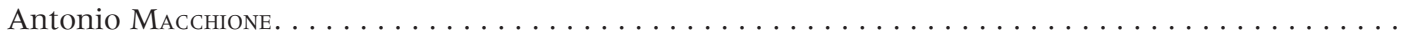

